\title{
Performance of Turbo-coded OFCDM Systems with Soft MCI Cancellation
}

\author{
Yiqing Zhou \\ Dept. of Electrical and Electronic Engineering \\ University of Hong Kong \\ Pokfulam Road, Hong Kong \\ yqzhou@eee.hku.hk
}

\author{
Jiangzhou Wang \\ Dept. of Electronics \\ University of Kent \\ Canterbury, Kent, CT2 7NT, United Kingdom \\ j.z.wang@kent.ac.uk
}

\begin{abstract}
This paper investigates the performance of turbocoded orthogonal frequency and code division multiplexing (OFCDM) systems with soft multi-code interference (MCI) cancellation. In order to regenerate the soft interference signal, the conventional turbo decoding algorithm must be modified to provide log likelihood ratio (LLR) values for all coded bits. Based on the LLR outputs of turbo decoder, two soft decision functions are proposed, i.e., LLR-soft-decision and Gaussian-soft-decision functions. By means of computer simulations, it is shown that in a highly frequency selective channel, soft MCI cancellation and MMSE detection can significantly improve the performance of turbo-coded OFCDM systems. Two iterations in turbo decoding are sufficient for both hard and soft decision functions. The proposed soft decision functions outperform the hard decision function with various channel conditions and system parameters.
\end{abstract}

Keywords-OFDM; two dimensional spreading; interference cancellation; soft decision function

\section{INTRODUCTION}

In future wireless communications, a huge bandwidth is needed, especially in downlink. The orthogonal frequency and code division multiplexing (OFCDM) system is a promising candidate for the broadband downlink transmission [1]. Based on the orthogonal frequency division multiplexing (OFDM) technique, OFCDM systems are robust to the severe multipath interference in a broadband channel. Moreover, twodimensional (2-D) spreading, i.e., time and frequency domain spreading, is introduced in the OFCDM system to provide more flexibility to the system deployment.

The orthogonal variable spreading factor (OVSF) codes [2] are employed in the OFCDM system as the 2-D spreading codes. The user data rate can be increased by using multicode transmission. However, in a realistic broadband mobile channel, the code orthogonality will be distorted by the possible fast fading and more seriously, the frequency selectivity. Therefore, in the OFCDM systems, multicode interference (MCI) is unavoidable in the high speed transmission. In order to improve the system performance, MCI cancellation must be considered.

The basic idea of MCI cancellation is to use the decision signals of previous stage to regenerate MCI, then subtract MCI from the received signal. The decision function could be hard or soft. In [3], hard decisions are used for coded OFCDM systems. In order to improve the system performance with hard decisions, a soft decision function should be used to regenerate $\mathrm{MCI}$.

In this paper, a slow fading and highly frequency selective channel is considered. Equal gain combining (EGC) is employed with time despreading, while minimum mean square error (MMSE) combining is used in frequency despreading. In the concerned OFCDM system with quadrature phase shift keying (QPSK) modulation and turbo coding, soft data decisions are carried out on the decoding output. Since all coded bits are required to regenerate the MCI, a modified turbo decoding algorithm is proposed to provide the loglikelihood ratio (LLR) of all coded bits. Based on the LLR output, the optimal soft decision functions which minimize the decision errors are derived.

The rest of the paper is organized as follows. Section II describes the OFCDM system. The operation of soft MCI cancellation and MMSE detection is shown in Section III, where the optimum soft decision functions are derived. Then the performance of the turbo-coded OFCDM system with soft MCI cancellation and MMSE detection is evaluated in Section IV by computer simulations. Finally, conclusions are drawn in Section V.

\section{System Description}

\section{A. Transmitter}

The basic structure of the turbo-coded OFCDM system is illustrated in Fig. 1. It is assumed that the $\bar{k}^{\text {th }}$ data channel is the desired channel. The data bits are firstly turbo-encoded [4], then translated into QPSK symbols, and processed by a symbol interleaver. The interleaved data symbols are then serial-toparallel (S/P) converted into $N_{B}$ streams, modulating $M$ subcarriers after spreading. Each symbol is firstly spread into $N_{T}$ chips in time domain with the spreading code $C_{N_{T}}^{\left(\bar{k}_{T}\right)}=\left\{c_{N_{T}, 0}^{\left(\bar{k}_{T}\right)}, \cdots, c_{N_{T}, N_{T}-1}^{\left(\bar{k}_{T}\right)}\right\}$. Then, the time domain spread signal is duplicated into $N_{F}$ copies and multiplied by the frequency domain spreading code $C_{N_{F}}^{\left(\bar{k}_{F}\right)}=\left\{c_{N_{F}, 0}^{\left(\bar{k}_{F}\right)}, \cdots, c_{N_{F}, N_{F}-1}^{\left(\bar{k}_{F}\right)}\right\}$. Therefore, totally $N=N_{T} \times N_{F}$ spread chips per data symbol are obtained by means of the 2-D spreading. Totally $M=N_{B} N_{F}$ spread 
chips are obtained in frequency domain. These $M$ chips are interleaved so that the $N_{F}$ sub-carriers corresponding to the $N_{F}$ chips carrying the same data symbol are separated in the frequency band, experiencing different fading and providing maximum frequency diversity gain. After interleaving, the $M$ chips will be up-converted to $M$ sub-carriers and transmitted in parallel. Note that this up-conversion at the transmitter and the down-conversion at the receiver could be realized by an $M$ point IFFT and FFT block, respectively, in practical systems. The signals from the $K$ code channels and the pilot channel will be added together at the code multiplexer.

\section{B. Channel Model}

Under the assumption of perfect suppression of multipath by the guard interval, a parallel slow fading multichannel model is employed. The signal transmitted on each sub-carrier is multiplied by a complex fading factor $H_{m}(m=0, \cdots, M-1)$, then added by a complex additive white Gaussian noise $\eta_{m}(t)$ with power spectral density of $N_{0}$. The amplitude and phase of $H_{m}$ are assumed to be Rayleigh distributed with $E\left\{\left|H_{m}\right|^{2}\right\}=1$ and uniformly distributed in $[0,2 \pi]$, respectively. The correlation between any two channel fades $H_{m_{1}}$ and $H_{m_{2}}$ is introduced by the frequency separation $\Delta f_{m_{1}, m_{2}}=f_{m_{1}}-f_{m_{2}}$, given by

$\rho_{m_{1}, m_{2}}=E\left\{H_{m_{1}}^{*} H_{m_{2}}\right\}=\frac{1}{\sqrt{1+\left(\Delta f_{m_{1}, m_{2}} / \Delta f_{c}\right)^{2}}}$

where $(\cdot)^{*}$ stands for the conjugate operation and $\Delta f_{c}$ is the channel coherence bandwidth.

\section{Receiver}

Passing through the parallel multichannel, the received signal is firstly down-converted and then passes through the integrator. The resultant signal on the $m^{\text {th }}$ sub-carrier in the $i^{\text {th }}$ OFCDM symbol duration is given by

$r_{m, i}=\left(\sum_{k=0}^{K-1} \sqrt{P} d_{k, m, i} c_{N_{T}, i}^{\left(k_{T}\right)} c_{N_{F}, m}^{\left(k_{F}\right)}+\sqrt{\beta P} d_{P, m, i}\right) H_{m}+\eta_{m, i}$

where $P$ is the signal power, $\beta$ is the power ratio of the pilot channel to one data channel, $d_{k, m, i}$ is the modulated data symbol of the $k^{\text {th }}$ data channel on the $m^{\text {th }}$ sub-carrier in the $i^{\text {th }}$ OFCDM symbol duration, $d_{P, m, i}$ is the known QPSK symbol in the pilot channel with the all " +1 " spreading code, and $\eta_{m, i}$ is the noise term.

In a slow fading channel, using the orthogonally code multiplexed pilot channel, the channel fading on each subcarrier can be estimated by firstly despreading the pilot channel and then averaging the signals over the whole packet duration. Since the orthogonality in time domain between the pilot channel and data channels preserves at the receiver side, the estimated channel fading is interference-free and only corrupted by the channel noise. The estimated channel fading is denoted as $\hat{H}_{m}$. With the estimated fading factors, the signals are then combined in time domain with EGC technique. The weight of EGC for the $\bar{k}^{\text {th }}$ code on the $m^{\text {th }}$ subcarrier in the $i^{\text {th }}$ OFCDM symbol duration is given by

$\bar{\varphi}_{\bar{k}, m, i}=\frac{\hat{H}_{m}^{*}}{\left|\hat{H}_{m}\right|} \cdot c_{N_{T}, i}^{\left(\bar{k}_{T}\right)}=\varphi_{m} \cdot c_{N_{T}, i}^{\left(\bar{k}_{T}\right)}$

where $\varphi_{m}=\hat{H}_{m}^{*} /\left|\hat{H}_{m}\right|$.

It should be noted that except the desired code $\left\{C_{N_{T}}^{\left(\bar{k}_{T}\right)}, C_{N_{F}}^{\left(\bar{k}_{F}\right)}\right\}$, the rest $(K-1)$ 2-D codes can be divided into two subsets: $\Omega_{F}=\left\{\left\{C_{N_{T}}^{\left(\bar{k}_{T}\right)}, C_{N_{F}}^{\left(k_{F}\right)}\right\} \mid k_{F} \neq \bar{k}_{F}\right\}$ and $\Omega_{T}=\left\{\left\{C_{N_{T}}^{\left(k_{T}\right)}, C_{N_{F}}^{\left(k_{F}\right)}\right\} \mid k_{T} \neq \bar{k}_{T}\right\}$. In a slow fading channel, the orthogonality between the desired code and the code channels in $\Omega_{T}$ can be preserved. Thus, the output of the time domain combiner is only interfered by signals from the code channels in $\Omega_{F}$. Therefore, soft MCI cancellation is carried out after time domain despreading, given by

$r_{\bar{k}, m, 0}^{(s)}=\frac{1}{N_{T}} \sum_{i=0}^{N_{T}-1} r_{m, i} \bar{\varphi}_{\bar{k}, m, i}-Q_{\bar{k}, m, 0}^{(s)}$

where $Q_{\bar{k}, m, 0}^{(s)}$ is the regenerated soft MCI for the interested data signal on the desired code channel and the superscript of $s$ stands for the $s^{\text {th }}$ stage. The resultant signals are then combined in frequency domain with MMSE technique. The MMSE weight at the $s^{\text {th }}$ stage is given by

$\bar{\omega}_{\bar{k}, m}^{(s)}=\frac{E\left\{\sqrt{P} d_{\bar{k}, m, 0}\left(r_{\bar{k}, m, 0}^{(s)}\right)^{*}\right\}}{E\left\{\left|r_{\bar{k}, m, 0}^{(s)}\right|^{2}\right\}}=\frac{P H_{m}^{*} \varphi_{m}^{*}}{E\left\{\left|r_{\bar{k}, m, 0}^{(s)}\right|^{2}\right\}} c_{N_{F}, m}^{\left(\bar{k}_{F}\right)}=\omega_{\bar{k}, m}^{(s)} c_{N_{F}, m}^{\left(\bar{k}_{F}\right)}$

The estimation of $\bar{\omega}_{\bar{k}, m}^{(s)}$ can be carried out as described in [3].

The output of the MMSE combiner for the desired code channel is given by

$$
\begin{aligned}
y_{\bar{k}, 0,0}^{(s)} & =\sum_{m=0}^{N_{F}-1} r_{\bar{k}, m N_{B}, 0}^{(s)} \bar{\omega}_{\bar{k}, m N_{B}}^{(s)} \\
& =\sqrt{P} d_{\bar{k}, 0,0} \sum_{m=0}^{N_{F}-1}\left(H_{m N_{B}} \varphi_{m N_{B}}\right) \omega_{\bar{k}, m N_{B}}^{(s)}+M C I_{\bar{k}, 0,0}^{(s)}+\eta_{\bar{k}, 0,0}
\end{aligned}
$$

where $y_{\bar{k}, 0,0}^{(s)}$ corresponds to the $0^{\text {th }}$ data symbol in both frequency domain and time domain, the first term on the right hand side of (6) is the desired signal, $M C I_{\bar{k}, 0,0}^{(s)}$ is the residual MCI, and $\eta_{\bar{k}, 0,0}$ is the noise term. In order to get the unbiased signal, $y_{\bar{k}, 0,0}^{(s)}$ should be normalized by the factor $\sum_{m=0}^{N_{F}-1}\left(H_{m N_{B}} \varphi_{m N_{B}}\right) \omega_{k, m N_{B}}^{(s)}$. After normalization, the resultant signals are then demodulated and turbo decoded to recover the transmitted information bit sequence.

\section{SOFT MCI CANCELLATION AND MMSE DETECTION}




\section{A. Modified Max_Log_Map Turbo Decoder}

In a conventional turbo decoder, only the information bits (or systematic bits) are concerned. Therefore, the decoding algorithm is derived to provide LLR of each systematic bit. As a result, only systematic bits are recovered after turbo decoding. However, in the soft interference cancellation, soft data replica must be regenerated. Therefore, all coded bits should be recovered. Using a similar process as the decoding algorithm in [5], the conventional turbo decoding algorithm can be modified to provide the LLR of both systematic and parity bits.

\section{B. Optimal Soft Decision Functions}

At the output of the turbo decoder, the LLRs of systematic bits and parity bits are combined into one LLR stream, which is denoted as $\Lambda_{\bar{k}, l}^{(s)}, l=0,1, \cdots, 2 M_{D} N_{B}-1$ for the $\bar{k}^{\text {th }}$ data channel at the $s^{\text {th }}$ stage, where $M_{D}$ is the number of data symbols transmitted in one packet duration on each subcarrier. The soft data decision is given by $b_{\bar{k}, l}^{(s)}=f_{\text {dec }}^{(s)}\left(\Lambda_{\bar{k}, l}^{(s)}\right)$, where $f_{\text {dec }}^{(s)}(x)$ is the soft decision function and can be found by minimizing the conditional mean square decision error (MSDE) $E\left\{\left.\left|b_{\bar{k}, l}-f_{\text {dec }}^{(s)}\left(\Lambda_{\bar{k}, l}^{(s)}\right)\right|\right|^{2} \mid \Lambda_{\bar{k}, l}^{(s)}\right\}$, where $b_{\bar{k}, l}= \pm \sqrt{P / 2}$ is the transmitted coded bit before QPSK modulation. By differentiating the conditional MSDE with respect to $f_{d e c}^{(s)}(x)$ and letting the differentiation be zero, one obtains

$f_{\text {dec }}^{(s)}\left(\Lambda_{\bar{k}, l}^{(s)}\right)=E\left\{b_{\bar{k}, l} \mid \Lambda_{\bar{k}, l}^{(s)}\right\}=\sum_{b_{\bar{k}, l}= \pm \sqrt{P / 2}} b_{\bar{k}, l} \operatorname{Pr}\left\{b_{\bar{k}, l} \mid \Lambda_{\bar{k}, l}^{(s)}\right\}$

It can be seen that the soft decision function $f_{d e c}^{(s)}(x)$ is decided by the statistic property of the turbo decoding output $\Lambda_{\bar{k}, l}^{(s)}$. Based on different interpretations of $\Lambda_{\bar{k}, l}^{(s)}$, two different soft decision functions can be obtained. Firstly, the physical meaning of $\Lambda_{\bar{k}, l}^{(s)}$ is exploited, i.e., $\Lambda_{\bar{k}, l}^{(s)}$ is the LLR of coded bits. Using the definition of LLR, a soft decision function called $L L R$-soft-decision function will be derived. On the other hand, regardless of the original definition, the final turbo decoding output $\Lambda_{\bar{k}, l}^{(s)}$ can be approximated as a BPSK modulated signal passing through an AWGN channel [4]. Based on this assumption, the probability density function of $\Lambda_{\bar{k}, l}^{(s)}$ can be obtained and another soft decision function called Gaussian-soft-decision function will be derived.

\section{B.1. LLR-soft-decision function}

First of all, the original definition of LLR is exploited to obtain $f_{d e c}^{(s)}(x)$. The turbo decoder output $\Lambda_{\bar{k}, l}^{(s)}$ is actually a scaled LLR, given by

$\Lambda_{\bar{k}, l}^{(s)}=\frac{\sigma_{\bar{k}, \xi}^{2}(s)}{\sqrt{2 P}} \cdot \log \frac{\operatorname{Pr}\left(b_{\bar{k}, l}=\sqrt{P / 2} \mid \text { observation }\right)}{\operatorname{Pr}\left(b_{\bar{k}, l}=-\sqrt{P / 2} \mid \text { observation }\right)}$ where $\sigma_{\bar{k}, \xi}^{2}(s)$ is the noise variance in the normalized detection output. Since

$$
\left\{\begin{array}{l}
\operatorname{Pr}\left(b_{\bar{k}, l}=\sqrt{P / 2} \mid \text { observation }\right)=\frac{e^{\Lambda_{\bar{k}, l}^{(s)} \sqrt{2 P} / \sigma_{k, \xi}^{2}(s)}}{1+e^{\Lambda_{\bar{k}, l}^{(s)} \sqrt{2 P} / \sigma_{\bar{k}, \xi}^{2}(s)}}, \\
\operatorname{Pr}\left(b_{\bar{k}, l}=-\sqrt{P / 2} \mid \text { observation }\right)=\frac{1}{1+e^{\Lambda_{\bar{k}, l}^{(s)} \sqrt{2 P} / \sigma_{\bar{k}, \xi}^{2}(s)}}
\end{array}\right.
$$

the optimal soft decision function can be derived as

$$
\begin{aligned}
f_{\text {dec }}^{(s)}\left(\Lambda_{\bar{k}, l}^{(s)}\right) & =\sqrt{P / 2} \cdot \frac{e^{\Lambda_{\bar{k}, l}^{(s)} \sqrt{2 P} / \sigma_{\bar{k}, \xi}^{2}(s)}}{1+e^{\Lambda_{\bar{k}, l}^{(s)} \sqrt{2 P}} / \sigma_{\bar{k}, \xi}^{2}(s)}-\sqrt{P / 2} \cdot \frac{1}{1+e^{\Lambda_{\bar{k}, l}^{(s)} \sqrt{2 P} / \sigma_{\bar{k}, \bar{s}}^{2}(s)}} \\
& =\sqrt{P / 2} \tanh \left(\frac{\sqrt{2 P}}{2 \sigma_{\bar{k}, \xi}^{2}(s)} \Lambda_{\bar{k}, l}^{(s)}\right)
\end{aligned}
$$

where $\tanh (x)=\frac{e^{x}-e^{-x}}{e^{x}+e^{-x}}$.

A data decision aided algorithm is proposed to estimate $\sigma_{\bar{k}, \xi}^{2}(s)$. Firstly, at the output of the MMSE detection, a hard decision is made on the observed complex signal sequence $\left\{\begin{array}{l}-(s) \\ y_{\bar{k}, n, j}\end{array}\right\}$ of the concerned code channel, denoted as $\left\{\bar{d}_{\bar{k}, n, j}^{(s)}\right\}$. Then, the variance $\sigma_{\bar{k}, \xi}^{2}(s)$ is given by

$\sigma_{\bar{k}, \xi}^{2}(s)=E\left\{\left|\xi_{\bar{k}, n, j}^{(s)}\right|^{2}\right\} \approx \frac{1}{M_{D} N_{B}} \sum_{n=0}^{N_{B}-1} \sum_{j=0}^{M_{D}-1}\left|\bar{y}_{\bar{k}, n, j}^{(s)}-\bar{d}_{\bar{k}, n, j}^{(s)}\right|^{2}$

\section{B.2. Gaussian-soft-decision function}

According to the research on turbo codes [4], the decoding output $\Lambda_{\bar{k}, l}^{(s)}$ can be approximated as a BPSK modulated signal passing through an AWGN channel. The sign of the assumed BPSK signal is the same as that of $b_{\bar{k}, l}$ and the amplitude is $E_{\Lambda, \bar{k}}^{(s)}$. Therefore, $\Lambda_{\bar{k}, l}^{(s)}$ can be approximated by

$\Lambda_{\bar{k}, l}^{(s)}=\left(E_{\bar{k}, l}^{(s)} / \sqrt{P / 2}\right) b_{\bar{k}, l}+v_{\bar{k}, l}$

where $v_{\bar{k}, l}$ is the assumed AWGN noise with a variance of $\sigma_{\Lambda, \bar{k}}^{2}(s)$. Hence, the decision function is given by

$$
\begin{aligned}
f_{\text {dec }}^{(s)}\left(\Lambda_{\bar{k}, l}^{(s)}\right)= & \frac{\sum_{b_{\bar{k}, l}} b_{\bar{k}, l} e^{-\left(\Lambda_{\bar{k}, l}^{(s)}-\frac{E_{\Lambda, \bar{k}}^{(s)}}{\sqrt{P / 2}} b_{\bar{k}, l}\right)^{2} /\left(2 \sigma_{\Lambda, \bar{k}}^{2}(s)\right)}}{\sum_{b_{\bar{k}, l}} e^{-\left(\Lambda_{\bar{k}, l}^{(s)} \frac{E_{\Lambda, \bar{k}}^{(s)}}{\sqrt{P / 2}} b_{\bar{k}, l}\right)^{2} /\left(2 \sigma_{\Lambda, \bar{k}}^{2}(s)\right)}} \\
= & \sqrt{P / 2} \tanh \left(\frac{E_{\Lambda, \bar{k}}^{(s)}}{\sigma_{\Lambda, \bar{k}}^{2}(s)} \Lambda_{l, \bar{k}}^{(s)}\right)
\end{aligned}
$$

In practical systems, the parameters $E_{\Lambda, \bar{k}}^{(s)}$ and $\sigma_{\Lambda, \bar{k}}^{2}(s)$ can be estimated by 


$$
\left\{\begin{array}{l}
E_{\Lambda, \bar{k}}^{(s)} \approx \frac{1}{2 M_{D} N_{B}} \sum_{l=0}^{2 M_{D} N_{B}-1}\left|\Lambda_{\bar{k}, l}^{(s)}\right| \\
\sigma_{\Lambda, \bar{k}}^{2}(s) \approx \frac{1}{2 M_{D} N_{B}} \sum_{l=0}^{2 M_{D} N_{B}-1}\left|\Lambda_{\bar{k}, l}^{(s)}\right|^{2}-\left[E_{\Lambda, \bar{k}}^{(s)}\right]^{2}
\end{array}\right.
$$

\section{Simulation Results}

\section{A. Configurations}

Computer simulation results are presented in this section. The major system parameters are defined as follows. The system employs a 2-D spreading factor of $N=4 \times 16.48$ code channels are used for data transmission. The power ratio between the pilot channel and all data channels $\beta / K$ is set to 0.2 . A bandwidth of $100 \mathrm{MHz}$ is employed and totally $M=1024$ sub-carriers are used. In one packet duration, there are $N_{d}=48$ OFCDM symbols per sub-carrier. The information bits transmitted on each data channel are individually encoded by a turbo code of $1 / 2$ rate. As to the parallel channel model, according to the typical urban channel model, the delay spread of the multi-path channel is around $2.14 \mu \mathrm{s}$. Then $\Delta f_{c}$ can be obtained as $1 / 2.14=0.47 \mathrm{MHz}$. Hence, the correlation between adjacent sub-carriers carrying the same data symbol is $\rho_{0, N_{B}}=1 / \sqrt{1+\left[B /\left(N_{F} \Delta f_{c}\right)\right]^{2}}=0.075$ for $N_{F}=16$. At the receiver side, the modified Max_Log_MAP algorithm is employed in the turbo decoder. Finally, the effective information bit signal to noise ratio (SNR) is defined as $S N R_{b}=\frac{P N(1+\beta / K)}{2 R_{e f f} \sigma_{n}^{2}}$, where 2 is the number of bits in one QPSK symbol and $R_{e f f}$ is the effective code rate.

\section{B. Verification of Gaussian Assumptions for $\bar{y}_{\bar{k}, n, j}^{-(s)}$ and $\Lambda_{\bar{k}, l}^{(s)}$}

First of all, the Gaussian distribution assumptions of the variables $\bar{y}_{\bar{k}, n, j}^{(s)}$ and $\Lambda_{\bar{k}, l}^{(s)}$ are verified by simulations. Note that $\bar{y}_{\bar{k}, n, j}^{(s)}$ is the normalized MMSE detection output at the $s^{\text {th }}$ stage for the $\bar{k}^{\text {th }}$ code channel, where $n$ and $j$ stand for the positions of the recovered data symbol in time and frequency domains, respectively. Given $P=2$ and $S N R_{b}=5.5 \mathrm{~dB}$, the number of iterations in turbo decoding is set to two. The $0^{\text {th }}$ data code channel is considered. Using the same set of channel fading factors, additive channel noise and data symbols are generated randomly. A 7-stage soft MCI cancellation is employed. When the LLR-soft-decision function is employed, the normalized MMSE detection outputs $\left(\bar{y}_{0, n, j}^{(s)}\right)$ are recorded at each stage, while when the Gaussian-soft-decision function is used, the signals at the output of turbo decoder $\left(\Lambda_{0, l}^{(s)}\right)$ are recorded. Totally 100 packets are simulated. The parameters, i.e., $\sigma_{\bar{k}, \bar{\xi}}^{2}(s), E_{\Lambda, \bar{k}}^{(s)}$ and $\sigma_{\Lambda, \bar{k}}^{2}(s)$, are estimated as explained in Sec. III. The decoding output is then normalized by $E_{\Lambda, \bar{k}}^{(s)}$. Histograms are shown in Fig. 2(a) and (b) for the signals demodulated from all $\bar{y}_{0, n, j}^{(s)}$ at the $0^{\text {th }}$ and $7^{\text {th }}$ stage, respectively, and in Fig. 2(c) for normalized decoded signals. As a comparison, histograms are also plotted in Fig. 3 for Gaussian distributed variables with the estimated variances of $\sigma_{\bar{k}, \xi}^{2}(s)$ and $\sigma_{\Lambda, \bar{k}}^{2}(s)$. In Fig. 2(a) and (b), it can be seen that at both the $0^{\text {th }}$ and $7^{\text {th }}$ stages, the observed demodulated signals are concentrated on the possible transmitted signals, +1 and -1 . At the $0^{\text {th }}$ stage, the $\mathrm{MCI}$ and noise in the demodulated signals are serious, and the curve is relatively flat. As soft MCI cancellation goes on, at the $7^{\text {th }}$ stage, part of the MCI is cancelled out and the curve becomes sharp. In both cases, the histograms of demodulated signals are similar to those obtained from Gaussian variables with the variance of $\sigma_{\bar{k}, \xi}^{2}(s)$. Meanwhile, the histograms of the decoded signals in Fig. 2(c) show the same properties as those of the demodulated signals. However, compared to the demodulated signals, the decoded signals are much closer to the Gaussian distributed variables. Hence, from Fig. 2, it can be concluded that the assumptions of Gaussian distribution for $\bar{y}_{\bar{k}, n, j}^{(s)}$ and $\Lambda_{\bar{k}, l}^{(s)}$ are reasonable, and $\Lambda_{\bar{k}, l}^{(s)}$ can be more accurately modeled as Gaussian variables than $\bar{y}_{\bar{k}, n, j}^{(s)}$.

\section{System Performance}

The packet error rate (PER) and bit error rate (BER) (after decoding) of the turbo-coded OFCDM system are illustrated in Fig. 3 when Gaussian-soft-decision MCI cancellation and MMSE detection is used. Since BER curves behave similarly to PER curves, only the description of PER is presented. It can be seen that at the $0^{\text {th }}$ stage without MCI cancellation, the system performance is quite poor. The PER performance is almost flat as SNR increases. Due to the low correlation $\rho_{0,64}=0.075$, the MCI is serious and even the powerful turbo decoding cannot work in such a deteriorative environment. Although SNR increases, the system performance is limited by the MCI, and PER cannot be reduced. However, when soft MCI cancellation is introduced, the system performance can be enhanced considerably. The PER decreases as the number of stages increases due to the reduction of MCI in each stage, especially when SNR is large. The most significant reduction in PER is obtained from the $0^{\text {th }}$ stage to the $1^{\text {st }}$ stage, then the reduction becomes smaller as the number of stages increases. However, since the MCI in a channel with low correlation is severe, there is still considerable improvement in PER even at the $7^{\text {th }}$ stage. For example, at $S N R_{b}=5.5 \mathrm{~dB}$, the PER at the $7^{\text {th }}$ stage is around $20 \%$ lower than that at the $6^{\text {th }}$ stage. Therefore, in a highly frequency selective channel, the performance of the turbo-coded OFCDM system can be improved significantly by the soft MCI cancellation. The larger the number of stages, the lower the PER. With $\rho_{0,64}=0.075$, a 7-stage soft MCI cancellation can provide good performance.

In the turbo-coded OFCDM system, the number of iterations used in turbo decoding is an important parameter. Fig. 4 shows the system PER as a function of the number of iterations. It can be seen that using a 7-stage MCI cancellation, 
a sharp reduction in PER is observed from the $1^{\text {st }}$ iteration to the $2^{\text {nd }}$ iteration. Then, the PER curve tends to be flat after the $2^{\text {nd }}$ iteration. This is because for the hybrid detection with multiple stages, much MCI has been cancelled out, and only few iterations are needed. Moreover, it can be seen that for various values of iterations, the MCI cancellation using soft decision functions always outperforms the one using hard decision function, and the Gaussian-soft-decision function provides better performance than the LLR-soft-decision. In summary, two iterations are sufficient to provide good performance for the turbo-coded OFCDM system with either hard or soft MCI cancellation.

Given a time domain spreading factor $N_{T}$ of 8 and a system load of 0.875 , the effect of the frequency domain spreading factor, $N_{F}$, on the system performance is investigated in Fig. 5 for $S N R_{b}=5.5 \mathrm{~dB}$ and $\rho_{0,64}=0.075 \quad\left(\Delta f_{c}=0.47 \mathrm{MHz}\right)$. For comparison, another set of curves (dashed) are also shown in Fig. 5 using higher correlation $\rho_{0,64}=0.564\left(\Delta f_{c}=4.27 \mathrm{MHz}\right)$ at higher $S N R_{b}=8.0 \mathrm{~dB}$. For both small and large correlation, it can be seen that for $N_{F}=1$, the system performance is not acceptable for either hard or soft decisions. In spite of no MCI, the input to the turbo decoder is too noisy for the decoder to work due to no frequency diversity gain. When $N_{F}$ is two, the PER reduces a little due to the frequency diversity gain. As $N_{F}$ is increased further, the system performance improves significantly. This is because although MCI in frequency domain increases, the frequency diversity gain also gets larger with increasing $N_{F}$. With MCI cancellation, the gain from diversity overcomes the loss from increased MCI. Therefore, the SNR of the input signal to turbo decoding improves, the decoder works effectively and the system performance is enhanced. The frequency diversity gain is saturated for large correlation when $N_{F}$ is large (i.e., $N_{F}=16$ ). However, the system performance can still be improved significantly with increasing $N_{F}$ for small channel correlation even when $N_{F}$ is large. This is because for large correlation of $\rho_{0,64}=0.564$, when $N_{F}$ increases from 16 to $32, \rho_{0, N_{B}}$ increases from 0.564 to 0.807 . Hence, the frequency diversity gain is saturated with the high $\rho_{0, N_{B}}$. However, for low correlation of $\rho_{0,64}=0.075, \rho_{0, N_{B}}$ is 0.15 for $N_{F}=32$. With this low correlation and MCI cancellation, there is still considerable frequency diversity gain and the system performance can be improved significantly.

\section{CONCLUSIONS}

The performance of the turbo-coded OFCDM system has been investigated with soft MCI cancellation in this paper. Based on the likelihood output of turbo decoding, two softdecision functions, i.e., LLR-soft-decision and Gaussian-softdecision, are proposed. The following conclusions are drawn:

1) Using Gaussian-soft-decision function as an example, soft MCI cancellation can improve the system performance significantly in a highly frequency selective channel. Gaussian function outperforms LLR function;

2) To carry out soft interference regeneration for the MCI cancellation, the conventional turbo decoding algorithm should be modified to decode both systematic and parity bits. Furthermore, for both hard and soft decision functions, two iterations are sufficient in the turbo decoding to provide stable performance;

3) Although frequency diversity gain is saturated for large channel correlation when $N_{F}$ is large, the gain increases further with increasing $N_{F}$ for small channel correlation even when $N_{F}$ is large.

\section{REFERENCES}

[1] H. Atarashi and M. Sawahashi, "Investigation of inter-carrier interference due to Doppler spread in OFCDM broadband packet wireless access," IEICE Trans. Commun., Special Issue on Software Defined Radio Technologies and Its Applications, vol. 86-B, 2002SRP28, pp. 291-299, Jan. 2003.

[2] F. Adachi, M. Sawahashi, and H. Suda, "Wideband DS-CDMA for next-generation mobile communications systems," IEEE Commun. Magazine, vol. 36, no. 9, pp. 56-69, Sept. 1998.

[3] Y. Zhou, J. Wang, and M. Sawahashi, "Downlink transmission of broadband OFCDM systems---Part III: Turbo-coded", To appear in $J S A C$, the issue on "The Next Generation CDMA Technologies".

[4] C. Berrou and A. Glavieux, "Near optimum error correcting coding and decoding: Turbo-Codes (1)," in Proc. IEEE ICC'93, pp. 1064-1070, May 1993.

[5] A. Wom, P. Hoeher, and N. When, "Turbo-Decoding without SNR estimation,” IEEE Commun. Letter, Vol.4, pp. 193-195, June 2000.

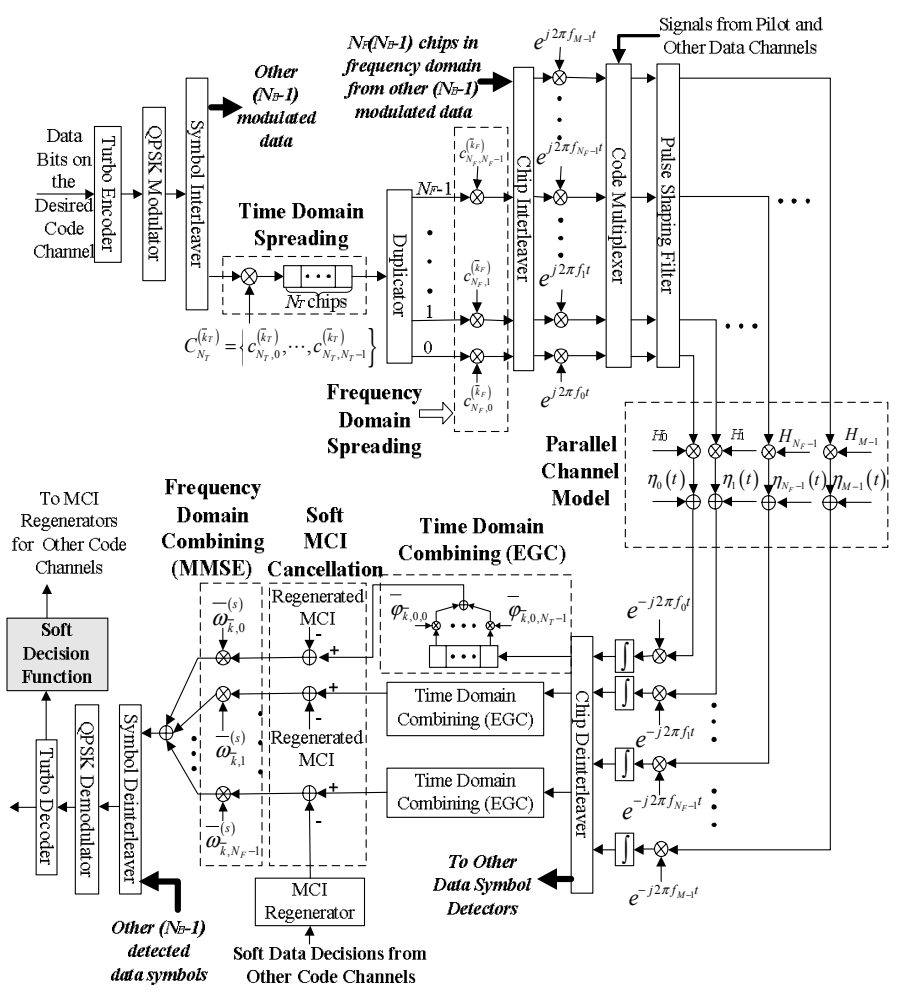

Figure 1. Basic structure of the OFCDM system 

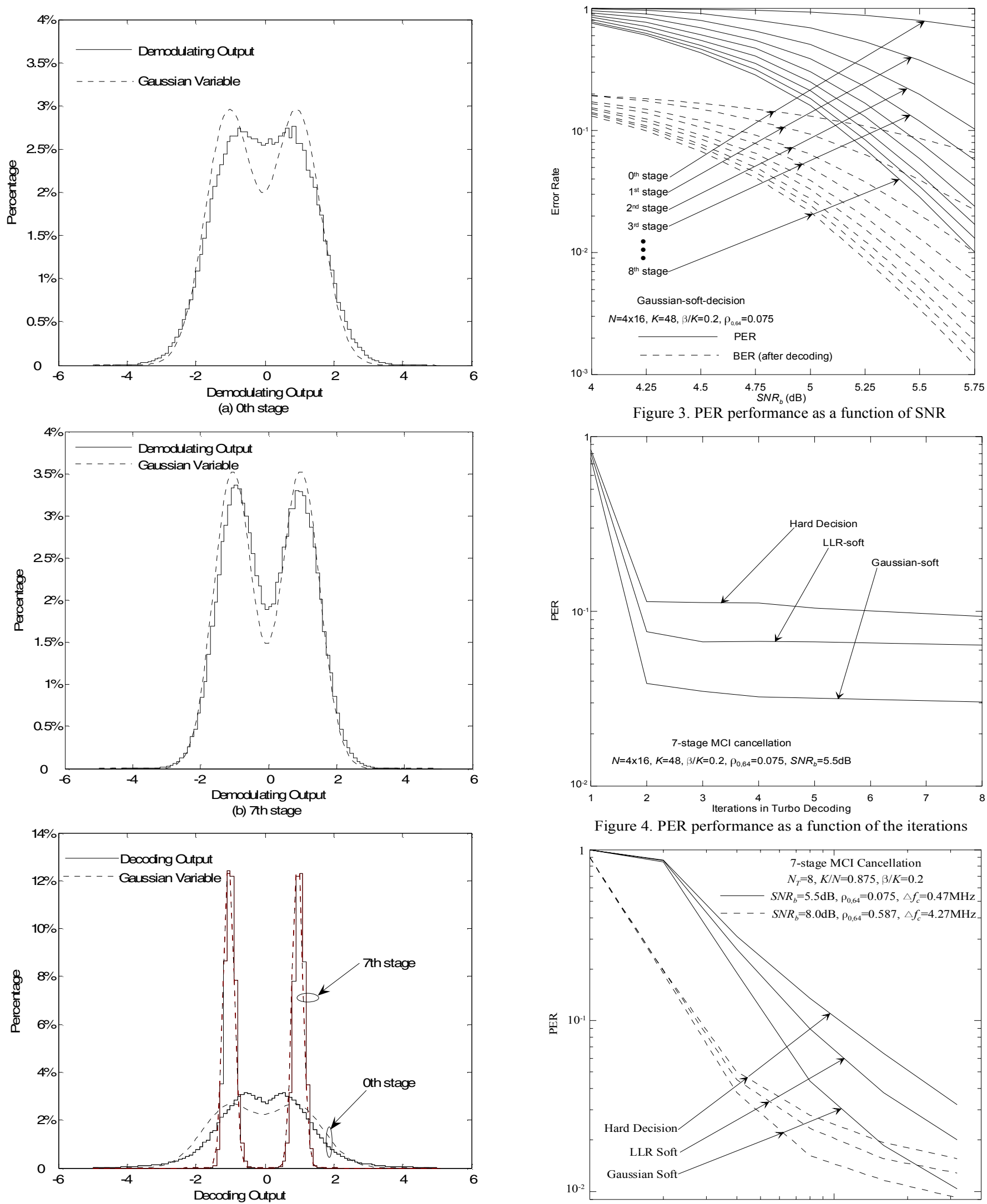

(c)

Figure 2. Histograms of observed signals

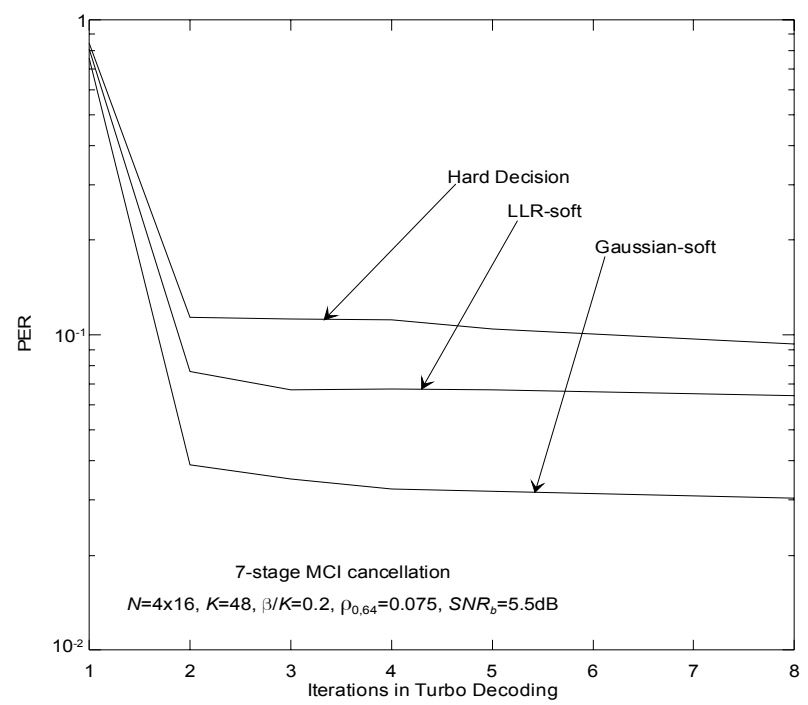

Figure 4. PER performance as a function of the iterations

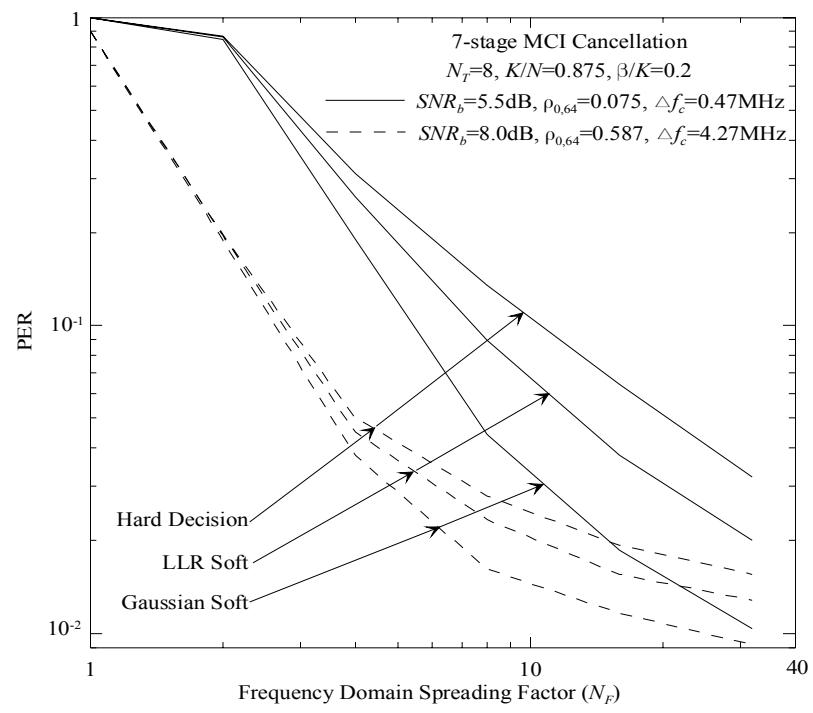

Figure 5. Effect of frequency domain spreading factor 